\title{
Functional Genetic Variants of PPARy and PPARa Encoding Peroxisome Proliferator-Activated Receptors and Susceptibility to Ischemic Stroke in Chinese Han Population
}

\author{
Yeqing Tong a, b, d Li Cai ${ }^{b, c}$ ShengHong Han ${ }^{a, b, d}$ Qing Lu ${ }^{b}$ Xuhua Guan ${ }^{a}$ \\ Xiaoxv Ying ${ }^{b}$ Shuangyi Hou ${ }^{a}$ Fanxian Zhan $^{a}$ Jin-quan Chengd, e Jiafa Liu ${ }^{a}$ \\ ${ }^{a}$ Hubei Center for Disease Control and Prevention, ${ }^{b}$ School of Public Health, Tongji Medical College, Huazhong \\ University of Science and Technology, and ' Wuhan Center for Disease Control and Prevention, Wuhan, ${ }^{\mathrm{d} K e y}$ \\ Laboratory of Molecular Biology of Guangdong Province, Shenzhen Center for Disease Controls and Prevention, \\ Shenzhen, and ' School of Public Health, University of Hong Kong, Hong Kong, PR China
}

\section{Key Words}

Ischemic stroke · Functional variants · Peroxisome

proliferator-activated receptors $\cdot$ Han population

\begin{abstract}
Background: PPARY and PPARa belong to a receptor family of ligand-activated transcription factors involved in the regulation of inflammation, cellular glucose uptake, protection against atherosclerosis and endothelial cell function. Through these effects, they might be involved with the ischemic stroke (IS). Methods: One thousand two hundred ninety-six subjects from the Chinese Han Population were chosen to assess the nature of the functional polymorphisms of PPARs and any links with IS. Multivariate logistic regression analysis was used to examine the association between PPARY and PPARa genotypes and a diagnosis of IS. Results: Pro/Ala carriage may be associated with the decreased risk
\end{abstract}

of IS in Hans (OR 0.542, 95\% Cl 0.346-0.850). The 162Val allele frequency at the DNA-binding region of PPARa was extremely rare in Chinese Han population. Conclusions: PPARY 12Pro/Ala resulting in an amino acid exchange in $\mathrm{N}$-terminal sequence may be an independent protective factor for IS in the Chinese Han population. However, more populations are warranted to validate our findings.

(c) 2015 S. Karger AG, Base

\section{Introduction}

Ischemic stroke (IS) is a major cause of morbidity and mortality in many developed and developing countries. High-density lipoprotein, homocysteine, troponin and fatty acid-related chronic low-grade inflammation and activation of the innate immune system are closely involved in the pathogenesis of IS [1-5]. Peroxisome pro-
Y.T., L.C., S.H., Q.L. and X.G. contributed equally to this work. 
liferator-activated receptor $\gamma$ and $\alpha$ (PPAR $\gamma$ and PPAR $\alpha)$ play key roles in the regulation of the inflammation, adipocyte differentiation and lipid biosynthesis [6]. Through these effects, PPAR $\gamma$ and PPARa might play crucial roles in the development and progression of IS. It was proposed that Leu162Val polymorphism and PPAR $\gamma$ Pro12Ala were associated with type 2 diabetes, atherosclerosis. However, their roles for IS have not yet been investigated.

In this study, we screened 648 patients with clinically definite IS and 648 age-, sex-, ethnically matched healthy controls from the Chinese Han subject panel for studying the association of PPAR $\alpha$ Leu162Val and PPAR $\gamma$ Pro12Ala polymorphisms with IS.

\section{Methods}

Our study was approved by the local Institutional Review Board. A total of 648 typical Uyghur IS subjects were enrolled into the case-control study. The diagnosis of IS was established according to the World Health Organization, and Trial of Org 10172 in Acute Stroke Treatment (TOAST) classification. The stroke subtypes were evaluated using the modified TOAST classification in total. Stroke was defined as a sudden onset of nonconvulsive and focal neurological deficit persisting for $24 \mathrm{~h}$. Large vessel was diagnosed if the patient had the characteristics of infarction due to low flow or artery-to-artery embolism in the presence of disease in the ipsilateral arterial tree (extracranial or intracranial segments of carotid or vertebrobasilar arteries, or proximal middle cerebral arteries) and CT scan or MRI performed within 7 days of onset showed either an area of infarction in the territory of a large vessel compatible with the clinical symptoms or hemodynamically significant stenosis. Small vessel was diagnosed if the patient had one of the characteristic clinical lacunar syndromes and CT scan or MRI showed either normal or a deep focal infarction in the brain stem or subcortical region with a diameter of $<1.5 \mathrm{~cm}$. A cardioembolic condition was diagnosed if the patient had the clinical characteristics of abrupt non-progressive onset, palpitations at onset, decreased consciousness at onset or hemianopia without hemiparesis and magnetic resonance angiography, computerized tomographic angiography, CT scan or MR diffusion-weighted imaging showed either a risk cardioembolic source in a region compatible with the clinical symptom or some embolisms located in other organs. The undetermined was defined as a kind that could not be classified as the previous 3 subtypes. Concurrently, 648 age-, gender- and ethnically matched normal healthy controls were randomly selected. Subjects with a history of stroke, Alzheimer's disease, brain aneurysm and Parkinson's disease were excluded from the controls group. An epidemiological investigation was performed with a standardized questionnaire to record epidemiological features.

Genotyping of PPARa Leu162Val and PPAR $\gamma$ Pro12Ala was performed by TaqMan SNP genotyping assays using ABI 7500 (Applied Biosystems, Foster City, Calif., USA). Multivariate logistic regression model analysis was used to examine the association between Pro12Ala genotypes and a diagnosis of IS.

PPARs Polymorphism and IS

\section{Results}

The characteristics of patients and controls are summarized in online supplementary table 1 (for all online suppl. material, see www.karger.com/doi/10.1159/000442306). The stroke subtypes were evaluated using the modified TOAST classification in total (large vessel [7.7\%], small vessel [36.7\%], cardioembolic [13.9\%], and undetermined [41.7\%]). TC, FBG, WHR, hypertension, history of heart diseases, and negative events may increase the risk of IS, with a trend for alcohol drinking, tea drinking and HDL to be protective factors for IS. In the additional multivariate logistic regression analysis, hypertension, diabetes, smoking, tea drinking, and WHR were also found to be independent predictors of IS among the Han population (online suppl. table 2).

Table 1 shows the distributions of the genotypes and allelic frequencies of PPARa Leu162Val and PPAR $\gamma$ Pro12Ala polymorphisms. There were no statistically significant difference in the distribution of PPARa Leu162Val polymorphism between the patients and controls in the Han ethnic group ( $\mathrm{p}>0.05)$. The Val allele frequencies at the 162 DNA-binding domain of PPARa were extremely low in the Chinese Han population. However, there were significant differences in the distribution of Pro12Ala polymorphism between the patients and controls in the Han group $(\mathrm{p}<0.01)$. The PPAR $\gamma$ Pro/Ala polymorphism was Hardy-Weinberg equilibrium in Han ethnic groups ( $\mathrm{p}>0.05)$. After adjustment for confounders, the PPAR $\gamma$ Ala allele was associated with a decreased risk of IS, with an overall OR of 0.542 and 0.555 when assuming Additive and Dominant models of inheritance, respectively (all $\mathrm{p}<0.01$ ). Assuming disease prevalence between 0.5 and $1 \%$, power analysis indicates that our sample size can reach $>75 \%$ power to detect Pro12Ala with the concluded genotypic ORs at the nominal Type I error rate $<0.05$ under Additive and Dominant genetic models.

Figure 1A compares the PPARa Leu162Val genotype frequency distribution between different ethnic groups based on the published research data and present study [7-10]. The PPARa 162Leu/Val genotype was more frequent in the Danish, German, Czech, Tunisia, and Brazil population than among the Asian Hans and Japanese (all $\mathrm{p}<0.05$ ). The PPAR $162 \mathrm{Val} / \mathrm{Val}$ genotype was more frequent only in German and Tunsia population than in Hans and Japanese (all $\mathrm{p}<0.05)$. The PPARa 162Leu/Val genotype was absent in Hans and the Japanese. Meanwhile, the PPARa 162Val/Val genotype was absent in Hans, Japanese, Germans and Brazils. Furthermore, we also analyzed the association of BMI, WHR, FBG of IS 
Table 1. PPARa Leu162Val and PPAR $\gamma$ Pro12Ala genotype and allele distributions between cases and controls in the Chinese Han population

\begin{tabular}{|c|c|c|c|c|c|c|}
\hline & \multicolumn{2}{|l|}{ Han } & $\mathrm{p}$ value & Crude OR (95\% CI) & $\mathrm{p}$ value & Adjusted OR* (95\% CI) \\
\hline Leu/Leu & 648 & 648 & & & & \\
\hline $\mathrm{Leu} / \mathrm{Val}$ & 0 & 0 & - & - & - & - \\
\hline $\mathrm{Val} / \mathrm{Val}$ & 0 & 0 & - & - & - & - \\
\hline Val & 0 & 0 & - & - & - & - \\
\hline MAF & 0 & 0 & - & - & - & - \\
\hline \multicolumn{7}{|l|}{ PPAR $\gamma(12)$} \\
\hline Pro/Pro & 606 & 580 & & & & \\
\hline Pro/Ala & 40 & 67 & 0.007 & $0.571(0.380-0.859)$ & & \\
\hline Ala/Ala & 2 & 1 & 1.000 & $1.914(0.173-21.167)$ & 1.000 & $1.915(0.172-21.170)$ \\
\hline
\end{tabular}

MAF $=$ Minor allele frequency. ${ }^{*}$ Multivariate logistic regression analysis after adjustment for sex, age, hypertension, diabetes, smoking, alcohol drinking, tea drinking, BMI, WHR.

Fig. 1. A The PPARa 162Leu/Val genotype frequency distribution between various populations. $\mathbf{\Delta}$ Compared with Han and Japanese populations on the Leu/Val genotype frequency; $\mathrm{p}<0.05$. Compared with Han and Japanese populations on the $\mathrm{Val} /$ Val genotype frequency; $p<0.05$. B The association of BMI, WHR, FBG of IS patients with Pro12Ala genotypes in the Han (a-c) ethnic group. $\Delta$ indicates $\mathrm{p}>0.05$ (Pro/Pro vs. Pro/Ala + Ala/Ala).

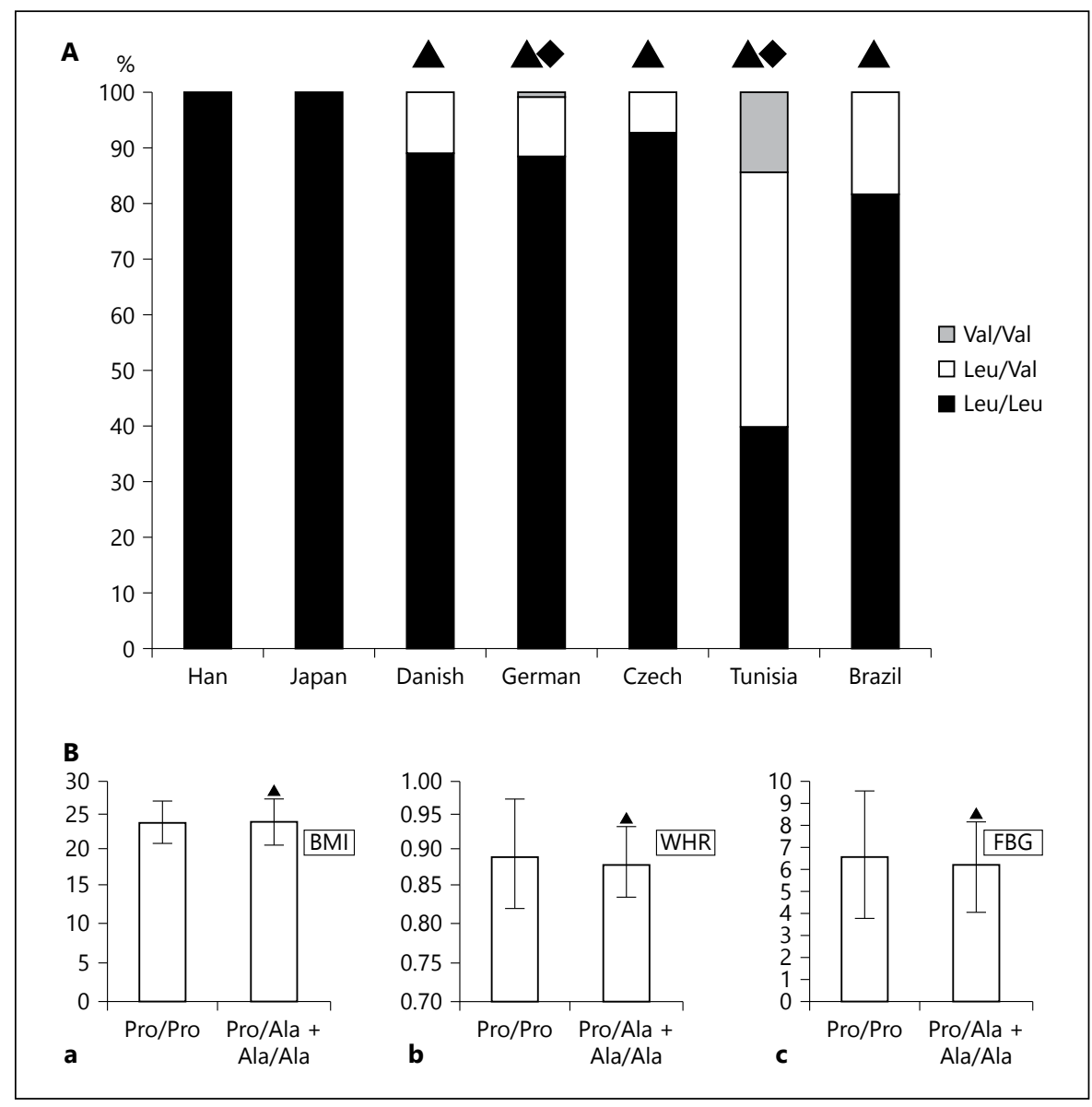


patients with Pro12Ala genotypes; however, we failed to find any significant association with any of these parameters in the Han population (all $\mathrm{p}>0.05$; fig. 1B).

\section{Discussion}

Taken together, we are the first to conclude that the $162 \mathrm{Val}$ allele frequency at the DNA-binding domain of human PPARa was extremely low in the Chinese Han population not similar to some European and African populations. The PPARa Leu162Val is unlikely to contribute significantly to a susceptibility to, or affect the progression of IS in Han population. Moreover, our findings lend support to the notion of an independent protective effect of the Ala allele of the PPAR $\gamma$ Pro12Ala on the risk of IS in the Han population.

However, our study also has certain limitations. Although the current study includes a relative large sample size in the Han population, rigorous methods used to diagnose IS, we used modified TOAST classification criteria which may be less comprehensive than ASCOD phenotyping [11] and lack of a validate population study to confirm our conclusions. Further explorations with larger, more ethnically diverse populations are warranted to better shed light on the functional properties of this receptor on the risk of IS, as well as the complicated pathophysiology mechanisms precisely. These findings may affect the diagnosis and treatment of IS.

\section{Acknowledgments}

This study was financially supported by research funds (No. 81302497 and81573237) from National Natural Science Foundation of China and Hubei Natural Science Foundation (No. 2013CFB056) and Hubei Province's outstanding medical academic leader program as well as China Postdoctoral Scientific Foundation (No. 2014M550394 and 2015T80807).

\section{References}

1 Wiseman S, Marlborough F, Doubal F, Webb DJ, Wardlaw J: Blood markers of coagulation, fibrinolysis, endothelial dysfunction and inflammation in lacunar stroke versus non-lacunar stroke and non-stroke: systematic review and meta-analysis. Cerebrovasc Dis 2014;37:64-75.

2 Corbett D, Nguemeni C, Gomez-Smith M: How can you mend a broken brain? Neurorestorative approaches to stroke recovery. Cerebrovasc Dis 2014;38:233-239.

3 Towfighi A, Arshi B, Markovic D, Ovbiagele B: Homocysteine-lowering therapy and risk of recurrent stroke, myocardial infarction and death: the impact of age in the VISP trial. Cerebrovasc Dis 2014;37:263267.

4 Lasek-Bal A, Kowalewska-Twardela T, Gąsior Z, Warsz-Wianecka A, Haberka M, Puz P, Ziaja $\mathrm{D}$ : The significance of troponin elevation for the clinical course and outcome of firstever ischaemic stroke. Cerebrovasc Dis 2014; $38: 212-218$.
5 López-Cancio E, Matheus MG, Romano JG, Liebeskind DS, Prabhakaran S, Turan TN, et al: Infarct patterns, collaterals and likely causative mechanisms of stroke in symptomatic intracranial atherosclerosis. Cerebrovasc Dis 2014;37:417-422.

6 Towfighi A, Ovbiagele B: Partial peroxisome proliferator-activated receptor agonist angiotensin receptor blockers. Potential multipronged strategy in stroke prevention. Cerebrovasc Dis 2008;26:106-112.

7 Sparsø T, Hussain MS, Andersen G, Hainerova I, Borch-Johnsen $\mathrm{K}$, Jørgensen $\mathrm{T}$, et al: Relationships between the functional PPARalpha Leu162Val polymorphism and obesity, type 2 diabetes, dyslipidaemia, and related quantitative traits in studies of 5799 middle-aged white people. Mol Genet Metab 2007;90:205-209.

8 Evans D, Aberle J, Wendt D, Wolf A, Beisiegel U, Mann WA: A polymorphism, L162V, in the peroxisome proliferator-activated receptor alpha (PPARalpha) gene is associated with lower body mass index in patients with noninsulin-dependent diabetes mellitus. J Mol Med (Berl) 2001;79:198-204.

9 Aldhoon B, Zamrazilová H, Aldhoon Hainerová I, Srámková P, Spálová J, Kunesová $\mathrm{M}$, et al: Role of the PPARalpha Leu162Val and PPARgamma2 Pro12Ala gene polymorphisms in weight change after 2.5year follow-up in Czech obese women. Folia Biol (Praha) 2010;56:116-123.

10 Dongiovanni P, Rametta R, Fracanzani AL, Benedan L, Borroni V, Maggioni P, et al: Lack of association between peroxisome proliferator-activated receptors alpha and gamma2 polymorphisms and progressive liver damage in patients with non-alcoholic fatty liver disease: a case control study. BMC Gastroenterol 2010;10:102.

11 Amarenco P, Bogousslavsky J, Caplan LR, Donnan GA, Wolf ME, Hennerici MG: The ASCOD phenotyping of ischemic stroke (updated ASCO phenotyping). Cerebrovasc Dis 2013;36:1-5. 